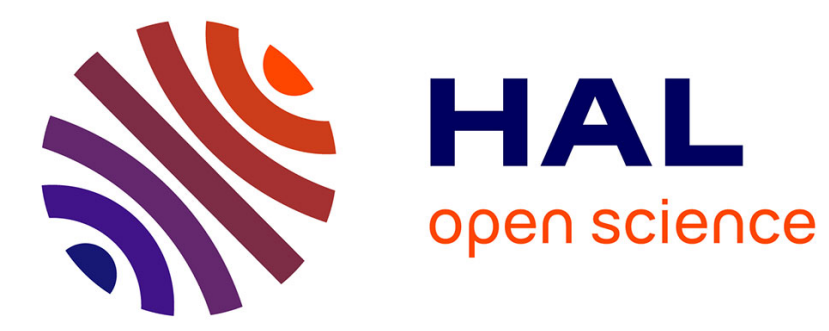

\title{
Finite element analysis of a fast electromagnetic actuator
}

Albert Foggia, Noël Burais, Laurent Krähenbühl, Alain Nicolas

\section{To cite this version:}

Albert Foggia, Noël Burais, Laurent Krähenbühl, Alain Nicolas. Finite element analysis of a fast electromagnetic actuator. IEEE Transactions on Magnetics, 1984, 20 (5), pp.1942 - 1944. 10.1109/TMAG.1984.1063332 . hal-00414132

\section{HAL Id: hal-00414132 \\ https://hal.science/hal-00414132}

Submitted on 8 Sep 2009

HAL is a multi-disciplinary open access archive for the deposit and dissemination of scientific research documents, whether they are published or not. The documents may come from teaching and research institutions in France or abroad, or from public or private research centers.
L'archive ouverte pluridisciplinaire HAL, est destinée au dépôt et à la diffusion de documents scientifiques de niveau recherche, publiés ou non, émanant des établissements d'enseignement et de recherche français ou étrangers, des laboratoires publics ou privés. 


\section{ABSTRACT}

The present paper is devoted to the modeliing of a fast acting linear electromagnetic actuator in which the mechanical and electrical time constants are of the same order of magnitude. The computation of the electromagnetic and the mechanical quantities must then be performed simultaneously. This paper describes the algorithm used to solve both the Maxwell's equations and the equation of motion.

\section{INTRODUCTION}

In many industrial applications, it is necessary to use linear electromechanical actuators in which the rapidity of displacement of the moving part is of prime importance. The geometry of such a device

is generally axisymmetric : it consists of an electromagnet of a solenoidal shape in which a cylindrical ferromagnetic yoke is allowed to have a linear motion. Usually, the excitation coil is associated with a fixed magnetic circuit.

Because of mechanical constraints, and for economic reasons, the magnetic circuits fixed and moving, may be solid. The designer of such circuits is then faced with a problem of eddy current distribution in addition to the magnetic saturation of the materials.

Regarding the excitation current in the coil, it is desirable that this current has a time variation adapted to the desired time response of the moving yoke. In our case, we assumed that the current is provided by an electronic current source, e.g. the current density in the excitation coil is assumed to be known at every time instant.

Finally, the mechanical time constant of this device is of the same order of magnitude as the electrical one. For this reason, the position of the moving yoke must be known with respect to time in order to take into account the mutual interaction between the magnetic quantities and the mechanical position and velocity of the yoke.

This is why, a step by step procedure has been developed that associates the electromagnetic equations and the equation of motion. Maxwell's equations have been solved using a finite element procedure in space and an implicit scheme for the time variation of the electromagnetic quantities. The equation of motion which is a second-order differential equation has been solved using a suitable time discretization scheme. The solution of the discretized quantities has been performed by means of an incomplete Choleski factorization associated with a conjugate gradient algorithm. The program has been implemented on an HP1000 computer.

The authors are with Departement d'Electrotechnique, ERA CNRS 908, Ecole Centrale de Lyon, BP 163, 69131 Ecully Cedex, FRANCE

\section{ELECTROMAGNETIC AND MOTION EQUATIONS}

The equations that govern the time and space variations of the electromagnetic quantities are the well-known Maxwell's equations. In the absence of displacement currents these equations read as follows :

$$
\begin{aligned}
& \operatorname{div} \vec{B}=0 \\
& \operatorname{cur} 1 \vec{H}=\vec{J}_{\text {ex }}+\vec{J} \\
& \operatorname{cur} 1 \vec{E}=-\frac{\delta \vec{B}}{\delta t} \\
& \vec{J}=\sigma \cdot \vec{E} \\
& \vec{B}=\mu \cdot \vec{H}
\end{aligned}
$$

using the following notations :

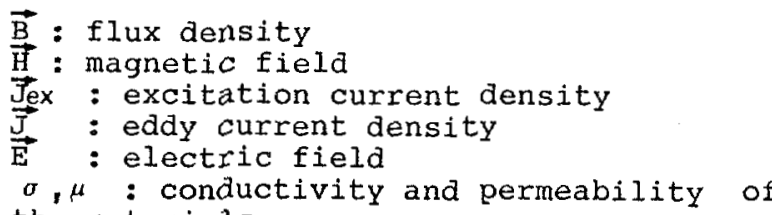
the materials

To solve these equations in the axisymmetric geometry of the actuator represented in figure 1 , it is convenient to use the magnetic vector potential defined by :

$$
\vec{B}=\operatorname{cur} 1 \vec{A}
$$

with the coulomb gauge

$$
\operatorname{div} \vec{A}=0
$$

When using the vector potential $\vec{A}$ as defined above, one obtains:

$$
\operatorname{Curl}\left(\frac{1}{\mu} \cdot \operatorname{Curl} \vec{A}\right)=-\sigma \cdot \frac{\delta \vec{A}}{\partial t}+J \overrightarrow{e x}
$$

which is the equation to be solved. In the particular case of an axisymmetric georetry, the vector potential $\vec{A}$ has one component only and this scalar function depends on 2 space variables $(r, z)$ and time t. Equation (4) then transforms into :

$\frac{\delta}{\delta z}\left(\frac{1}{\mu} \cdot \frac{\delta A}{\delta z}\right)+\frac{\partial}{\delta r}\left(\frac{1}{\mu} \cdot \frac{1}{r} \cdot \frac{\delta}{\delta r}(r \cdot A)\right)=\sigma \cdot \frac{\partial A}{\delta t}-J e x$

Equation (5) has to be solved taking into account the koundary conditions and in the reference frame of the fixed and the moving parts of the actuator. The position of the moving yoke is defined by the equation of motion given by :

$$
\mathrm{m} \ddot{\mathbf{z}}+\lambda \dot{z}+\mathrm{k} z=\mathrm{F}_{\mathrm{e}}-\mathrm{F}_{\mathrm{r}}
$$


where the following notations are used :

$$
\begin{aligned}
& m \text { : mass of the yoke } \\
& \lambda \text { : friction coefficient } \\
& k \text { : rigidity coefficient } \\
& \mathrm{F}_{\mathrm{e}} \text { : electric force } \\
& \mathrm{F}_{r} \text { : resistive force }
\end{aligned}
$$

Due to the low inertia of the yoke, the time constant of the motion is of the same order of magnitude as the time constant of the excitation coil. For this reason, equations (5) and (6) must be solved simultaneously at every time instant.

\section{DISCRETIZATION OF THE EQUATIONS}

In order to solve equations (5) and (6) it is necessary to adopt a numerical algorithm that takes into account the shape of the different parts of the actuator, the non linearity of the magnetic circuit and the time variations

of the different quantities. Moreover such a method must be stable with respect to time [1].

For all these reasons we have chosen a finite element method in space for equation (5); after discretization of the domain, the vector potential has been approximated using second-order triangular elements. In each element, the vector potential varies according to equation (7) :

$$
A(r, z, t)=\sum_{i}^{6} N_{i}(r, z) \cdot A_{i}(t)
$$

where $N_{i}(r, z)$ are second-order polynomials [2].

After assembling all the elementary equations, a differential system of equations is obtained which may be written as [1]:

$$
M \cdot A+N \cdot \frac{d A}{d t}=S
$$

Where $M$ and $N$ are $n \times n$ matrices; $n$ being the number of nodes of the finite element grid used. In equation ( 8$)$, the coefficients of the matrix $M$ depend on the permeability of the magnetic medium which makes equation (8) a non- linear one. In matrix $N$, the coefficients are constant ; they depend on the conductivity of the different media. $S$ is a column vector representing the excitation function due to the current flowing in the coil.

\section{SOLUTION OF THE DISCRETIZED EQUATIONS}

To solve equation (8), an implicit method has been used. Such a method has been proposed many years ago, and has proved to be very efficient in the computation of eddy currents in linear and non-linear media [1]. The implicit method consists of writting equation (8) at time $n \Delta t$

$$
M^{n} \cdot A^{n}+\frac{N}{\Delta t} \cdot\left(A^{n}-A^{n-1}\right)=S^{n}
$$

Solving with respect to $A^{n}$ leads to

$$
A^{n}=\left(M^{n}+\frac{N}{\Delta t}\right)^{-1} \cdot\left(S^{n}+\frac{N}{\Delta t} \cdot A^{n-1}\right)
$$

Equation (10) is non-linear and the solution is obtained by successive iterations. Provided the time step $\Delta t$ is small enough, the number of iterations is reduced. The solution of the system of equations, with a good approximation of the coefficients of $M$, has been obtained by means of an ICCG procedure [3]. Once equation (10) has been solved, the electromagnetic force exerted on the moving yoke is computed in order to solve the equation of motion (6). This is a second order equation with constant coefficients and may be written at time $n \Delta t$ as :

$$
m \ddot{z}_{n}=F_{e}^{n}-\lambda \dot{z}_{n}-k z_{n}-F_{r}
$$

the speed $\dot{z}_{n}$ and the position $z_{n}$ of the moving yoke may be approximated as

$$
\begin{aligned}
\dot{z}_{n}= & \dot{z}_{n-1}+(1-\gamma) \cdot \dot{z}_{n-1} \Delta t+\mathcal{\gamma} \cdot \ddot{z}_{n} \cdot \Delta t \\
z_{n}= & z_{n-1}+\dot{z}_{n-1} \Delta t+(1-\beta) \cdot \ddot{z}_{n-1} \cdot \frac{\Delta t^{2}}{2} \\
& +\beta \ddot{z}_{n} \cdot \frac{\Delta t^{2}}{2}
\end{aligned}
$$

Equations (11) to (13) may be solved simultaneously provided the electromagnetic force is known. $\beta$ and $\gamma$ are positive coefficients less than 1 [2].

In the program that has been written to simulate the actuator, all the quantities are computed at every time instant. starting with a given excitation current in the coil and the position of the yoke at rest, the vector potential is then computed which permits the computation of the force exerted on the yoke. The solution of the mechanical equation allows the position of the yoke to be known for the computation of the electromagnetic quantities for the next time interval.

In figure 2 , the computed results are presented. It is seen that the eddy currents behave like a viscosity and introduce a delay in the development of the force and the motion of the yoke is delayed accordingly.

\section{CONCLUSION}

In this paper, we have presented a method that allows Maxwell's equations to be solved simultaneously with an equation of motion. It has consisted of coupling the differential equation obtained after discretizing Maxwell's equations with finite elements in space. To this differential system, it has been necessary to add the mechanical equation. A suitable time discretization scheme has permitted to transform these differential equations into algebraic ones that has been solved using an ICCG method. The method described in this paper has been experimented on an electromagnetic linear actuator that has a fast time response, and has given satisfactory results. 
This method coupling mechanical and electrical equations may be extended to the modelling and simulation of other electromechanical devices in which the time constants of different nature are of the same order of magnitude.

\section{REFERENCES}

(1) A. Foggia, J.C. Sabonnadiere, P. Silvester, "Finite element solution of saturated travelling magnetic field problems", IEEE trans. on Power App. and Systems, Vol.PAS-94, May/June 1975, pp. $866-871$

(2) o.C. Zienkiewicz, The finite element in engineering science, Mc Graw Hill, London 1976

(3) J.A. Meijerink, H.A. Van der Vorst, "An iterative solution method for linear systems of which the coefficient matrix is a symmetric M-Matrix", Math. Comp. 31, 1977, pp. 148-162.

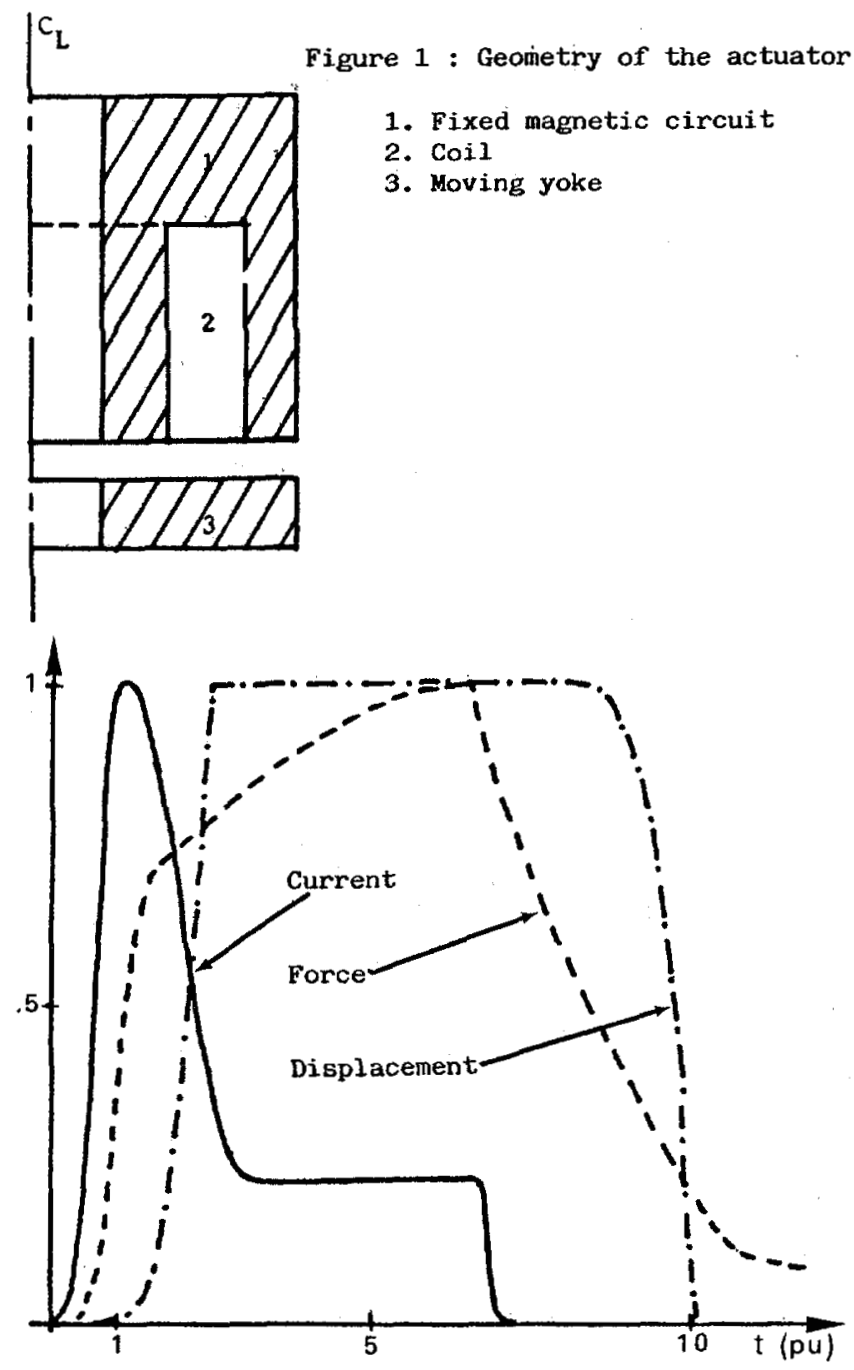

Figure 2 : Computed force and displacement 